Review began 08/12/2021 Review ended 10/01/2021 Published 10/05/2021

(c) Copyright 2021

Madan et al. This is an open access article distributed under the terms of the Creative Commons Attribution License CC-BY 4.0., which permits unrestricted use, distribution, and reproduction in any medium, provided the original author and source are credited.

\title{
Improving Ineffective Erythropoiesis in Thalassemia: A Hope on the Horizon
}

\author{
Ujjwal Madan $^{1}$, Himani Bhasin ${ }^{1}$, Pooja Dewan ${ }^{1}$, Jyotsna Madan ${ }^{2}$ \\ 1. Pediatrics, University College of Medical Sciences, Delhi, IND 2. Pathology, Super Speciality Pediatric Hospital and \\ Post Graduate Teaching Institute, Noida, Uttar Pradesh, IND
}

Corresponding author: Jyotsna Madan, drjyotsnamadan@yahoo.co.in

\begin{abstract}
Beta-thalassemia is an inherited hemoglobinopathy characterized by the impaired synthesis of beta-globin chains of hemoglobin leading to chronic hemolytic anemia. The mainstay of treatment for most patients remains regular blood transfusions and iron chelation. This conventional therapy has many limitations and challenges. Allogeneic hematopoietic stem cell transplant (HSCT) is the only available curative treatment but the availability of a suitable donor, financial constraints, and a need for specialist physicians can be limiting factors. Gene therapy is an upcoming curative therapeutic modality. An increased understanding of the underlying pathophysiology and molecular mechanisms of thalassemia has paved the way for novel pharmacological agents targeting ineffective erythropoiesis. These drugs act by decreasing transfusion requirements and hence decrease transfusion-related complications. The present review intends to provide an insight into the recent advances in pharmacological agents targeting ineffective erythropoiesis. Literature was searched and relevant articles evaluating newer drugs in thalassemia were collected from databases, including Pubmed, Scopus, Prospero, Clinicaltrials.gov, Google Scholar, and the Google search engine. We used the following keywords: thalassemia, novel, treatment, drugs, and ineffective erythropoiesis during the initial search. Relevant titles and abstracts were screened to choose relevant articles. Further, the full-text articles were retrieved and relevant cross-references were scanned to collect information for the present review.
\end{abstract}

Categories: Pediatrics, Hematology, Therapeutics

Keywords: jak-2 inhibitors, luspatercept, ruxolitinib, sotatercept, ferroportin inhibitors

\section{Introduction And Background}

Beta-thalassemia is an inherited hematological disorder caused by mutations in the beta-globin gene, which leads to insufficient beta-globin chain synthesis and, therefore, ineffective erythropoiesis [1]. The mainstay of treatment of beta-thalassemia includes life-long regular blood transfusions and iron chelation, resulting in severe complications compromising their overall survival [1]. Adverse effects associated with regular blood transfusions include systemic iron overload (cardiac, hepatic, endocrine, pulmonary dysfunction), infections, growth disturbances, and hemolytic and non-hemolytic blood transfusion reactions. Additionally, adverse effects of iron chelators used include gastrointestinal symptoms, arthralgia, skin rash, growth disturbances, visual defects, sensorineural hearing loss, and local infusion site reactions [1]. Although hematopoietic stem cell transplantation (HSCT) is curative, it is limited by the availability of human leukocyte antigen (HLA)-matched donors, the inherent risk of graft-versus-host disease (GVHD), and prohibitive costs. Recently, gene therapy using genetically modified autologous stem cells in thalassemia patients lacking a matched donor has rendered them transfusion-free, offering hope of cure [2].

Due to the limited availability of both these curative treatments, clinical studies have explored non-curative pharmacological treatment options such as erythropoiesis-stimulating agents that may prevent the complications associated with anemia, hemolysis, and iron overload [3-6]. Herein, we provide an insight into the recent advances in the drugs targeting ineffective erythropoiesis in beta-thalassemia. For the present review, we searched the literature and relevant articles evaluating newer drugs in thalassemia from databases, including PubMed, Scopus, Prospero, Clinicaltrials.gov, Google Scholar, and the Google search engine. The following keywords: thalassemia, novel, treatment, drugs, and ineffective erythropoiesis were used during the initial search. Relevant titles and abstracts were screened to choose relevant articles. Further, the full-text articles were retrieved and relevant cross-references were scanned to collect information for the present review.

\section{Review}

\section{Classification of newer pharmacological agents}

Several treatment modalities like drugs that act by increasing hemoglobin $(\mathrm{Hb}) \mathrm{F}$ (like hydroxyurea, azacytidine, thalidomide, and butyrates) or stimulate erythropoiesis (recombinant erythropoietin) have been tried in thalassemia with some success [3-6]. Among these drugs, thalidomide and hydroxyurea have been known to cause severe adverse effects, thus limiting their routine use in thalassemia treatment. Thalidomide 


\section{Cureus}

has been associated with leucopenia, birth defects, neuropathy, and increased risk of thrombosis. Hydroxyurea is known to cause myelosuppression. Recently, newer treatment modalities targeting ineffective erythropoiesis are also being explored to treat beta-thalassemia (Figure 1). Drugs targeting ineffective erythropoiesis can be classified under the following groups, viz, activin II receptor traps (Luspatercept and Sotatercept), Janus-associated Kinase 2 (JAK2) inhibitors (Ruxolitinib), drugs targeting iron metabolism (mini hepcidins, serine protease TMPRSS6 inhibitors, ferroportin inhibitors, and apotransferrin F), macrophage manipulation therapy, and heat shock proteins [7].

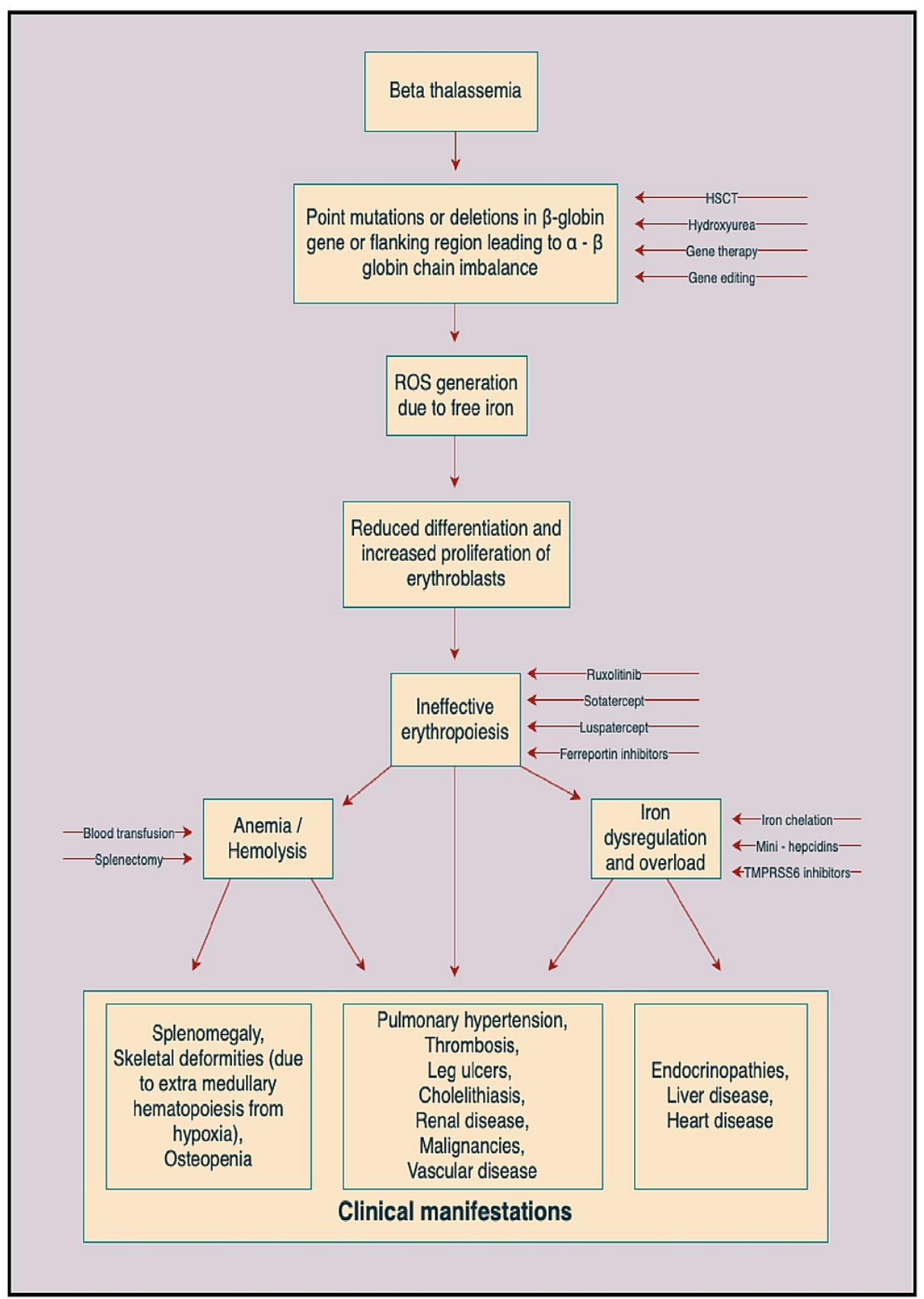

FIGURE 1: Mechanism of action of different treatment modalities in beta-thalassemia major

HSCT: hematopoietic stem cell transplant, ROS: reactive oxygen species, TMPRSS6: transmembrane protease serine 6

\section{Activin II receptor ligand traps}

Mechanism of action: Growth and differentiation factor 11 (GDF11), an activin belonging to the transformation growth factor- $\beta$ (TGF- $\beta$ ) family, plays a crucial role in erythropoiesis. GDF11 binds to activin type IIa receptor (ActRIIa) or type IIb receptors (ActRIIb) and induces the recruitment and phosphorylation 
of an activin type I receptor, which then phosphorylates the Smad2 and Smad3 intracellular signaling proteins. GDF11-ActRIIb-Smad2/3-dependent signaling is a key regulatory mechanism in proliferating erythroid precursors, as it controls their late-stage maturation. Growth differentiation factor 11 (GDF-11) has been postulated as the critical factor responsible for inhibiting late-stage erythroid differentiation and leading to ineffective erythropoiesis [8-9]. The activin II receptor ligand traps contain the extracellular domain of human activin receptor type IIb (ActRIIb), leading to reduced activin binding to the ActRIIb. Hence, they promote the terminal differentiation of erythroblasts with correction of maturation arrest. They reduce oxidative stress and the excess $\alpha$-globin aggregates, which are responsible for the ineffective erythropoiesis [10-11]. Activin receptor-II binding traps include two agents, Sotatercept (ACE011) and Luspatercept (ACE-536), which have shown promising results in thalassemia major.

Luspatercept

Luspatercept (ACE - 536) is a novel, soluble, recombinant fusion protein. Structurally, it has a modified extracellular domain of human activin receptor type IIb (ActRIIb) attached to the human IgG1 Fc domain [12-14].

Mechanism of action: Luspatercept enhances red blood cell (RBC) production by serving as a trap for ligands (belonging to the transforming growth factor-beta $\{$ TGF- $\beta$ \} superfamily) that inhibit late stages of erythropoiesis by diminishing Smad 2/3 intracellular signaling. Therefore, it promotes the differentiation and proliferation of late-stage erythroid precursors (normoblasts), improves hemoglobin concentration, and reduces the transfusion burden in thalassemia [12-16].

Pharmacokinetics: Luspatercept displays linear kinetics over doses of 0.2-1.25 mg/kg. A steady-state serum concentration is obtained after three subcutaneous doses $(1 \mathrm{mg} / \mathrm{kg}$ or $1.25 \mathrm{mg} / \mathrm{kg}$ ) administered every three weeks; a mean half-life $\left(\mathrm{t}_{1 / 2}\right)$ of approximately 11 days is seen. The absorption of the drug is unaffected by the site of injection [12]. The pharmacokinetics of luspatercept are independent of age, gender, race, ethnicity, hepatic or renal impairment, RBC transfusion burden, baseline serum albumin or erythropoietin levels, beta-thalassemia genotype ( $\beta_{0} / \beta_{0}$ vs. non - $\left.\beta_{0} / \beta_{0}\right)$, splenectomy, or the concomitant use of iron chelating agents [12-13].

Clinical trials: The BELIEVE study, a phase III trial (Clinical Trials.gov number NCT02604433), is a randomized, double-blind, placebo-controlled trial conducted on a total of 336 adult transfusion-dependent thalassemia (TDT) patients (receiving 6 to 20 units of packed red cells, with no transfusion-free period of $>35$ days, within 24 weeks before randomization). Participants were randomized to receive either luspatercept at a dose of 1 to $1.25 \mathrm{mg} / \mathrm{kg}$ or a placebo subcutaneously every three weeks for at least 48 weeks. The study's endpoints were an erythroid response, defined as the percentage of participants having a reduction in the transfusion burden of at least 33\% from the baseline (with a reduction of at least 2 RBC units) over a 12-week interval (from weeks 13-24). Secondary endpoints included a reduction in the transfusion burden of at least 33\% from baseline (with a reduction of at least 2 RBC units) during weeks 3748. They reported that the patients with a reduced transfusion burden of at least $33 \%$ from baseline (with a reduction of at least 2 RBC units) during weeks 13-24 were significantly higher in the luspatercept group as compared to the placebo group $(21.4 \%$ vs. $4.5 \%$, p $<0.001)$ along with a reduction in serum ferritin levels in the luspatercept group. Approximately $75 \%$ of patients had achieved at least a $33 \%$ reduction in transfusion burden during any 12-week period during the 48-week double-blind treatment with luspatercept [15-16].

Another open-label, dose-defining, non-randomized, uncontrolled, phase 2 study (Clinical Trials.gov number NCT01749540) was conducted in 31 non-transfusion dependent thalassemia (NTDT) patients (receiving $<4$ units of RBCs within 8 weeks prior to day 1 cycle 1 ) and 32 TDT patients (requiring $\geqslant 4$ units of RBCs every 8 weeks), with prior splenectomy or spleen size $<18 \mathrm{~cm}$ in the longest diameter by abdominal ultrasound. Luspatercept was administered subcutaneously every three weeks at a dose ranging from 0.2 to $1.25 \mathrm{mg} / \mathrm{kg}$ [17]. The erythroid response was defined as an increase in hemoglobin $(\mathrm{Hb})$ of $\geqslant 1.5 \mathrm{~g} / \mathrm{dL}$ from baseline for $\geqslant 2$ weeks (in the absence of RBC transfusions) for NTDT and $\geqslant 20 \%$ reduction in RBC transfusion burden compared to pretreatment in TDT patients over a period of 12 weeks. A mean increase in $\mathrm{Hb}$ from a baseline of $\geqslant 1.5 \mathrm{~g} / \mathrm{dL}$ over two weeks was noted in 18 (58\%) NTDT patients. In TDT patients, 26 (81\%) achieved a transfusion burden reduction of $\geqslant 20 \%$ over 12 weeks at a dose of 0.6 to $1.25 \mathrm{mg} / \mathrm{kg}$. The study recommended using luspatercept in a starting dose of $1 \mathrm{mg} / \mathrm{kg}$ (titration up to $1.25 \mathrm{mg} / \mathrm{kg}$ ) [13].

A phase 2, double-blind, randomized, placebo-controlled, multicenter study (BEYOND trial, Clinical Trials.gov number NCT03342404) is underway to determine the efficacy and safety of Luspatercept as compared to placebo in adults with NTDT (defined as receiving 0 to 5 units of RBCs during the 24 weeks and $\mathrm{RBC}$ transfusion-free for at least $\geqslant 8$ weeks prior to randomization). The study's primary endpoint is the estimation of the proportion of participants with an increase in $\mathrm{Hb}$ of $\geqslant 1.0 \mathrm{~g} / \mathrm{dl}$ from baseline over a 12-week interval (from weeks 13-24) in the absence of RBC transfusions [18].

Dosing: Luspatercept is available commercially as a white lyophilized powder in single-dose vials (Reblozyl ${ }^{\circledR}$, Acceleron Pharma - Celgene Corporation, $25 \mathrm{mg}$ and $75 \mathrm{mg} / \mathrm{vial}$ ) and is reconstituted with sterile water. The recommended dose for starting the therapy is $1 \mathrm{mg} / \mathrm{kg}$ subcutaneously once every three 
weeks. If there is no reduction in the patient's transfusion requirement after at least two consecutive doses (6 weeks), the dose may be escalated to a maximum dose of $1.25 \mathrm{mg} / \mathrm{kg}$. However, if still there is no response after nine weeks ( 3 doses) at a maximal dose or if the patient experiences unacceptable toxicity at any point of time, the drug must be discontinued [12]. No dose adjustment is needed in mild to severe hepatic insufficiency and mild to moderate renal disease [12].

Adverse events: In the BELIEVE trial, adverse effects (of all grades) were reported in $>5 \%$ of patients treated with luspatercept. The commonly reported side effects were back pain (27\%), upper respiratory tract infection (26\%), headache (26\%), bone pain (20\%), arthralgia (19\%), pyrexia (16\%), cough (14\%), fatigue (13\%), oropharyngeal pain (13\%), diarrhea (12\%), dizziness (11\%), myalgia (9\%), nausea (9\%), abdominal pain $(8 \%)$, hypertension (8\%), and hyperuricemia (7\%) [13]. Serious adverse effects $(3.6 \%)$ reported were cerebrovascular accidents and deep vein thrombosis (1\%). The patients must also be cautioned about the potential for immunogenicity, whereby the presence of neutralizing antibodies against luspatercept can decrease its serum concentration [12,15-16].

Safety: Currently, there are no absolute contraindications for luspatercept. However, caution must be exercised while prescribing luspatercept to patients with thromboembolic events, hypertension, pregnancy, lactation, and females of reproductive potential [12]. Animal studies have reported an increased risk of developing hematologic malignancies at doses of $10 \mathrm{mg} / \mathrm{kg}$, a dose approximately eight-fold higher than the maximum recommended human dose (MRHD). Fertility studies conducted in rats had reported effects on female (but not male) fertility at doses almost seven times higher than the MRHD. Female patients with betathalassemia being considered for Luspatercept treatment should undergo pregnancy testing before starting treatment and should also be advised to use contraception during treatment and three months after receiving the final dose. Lactating females should not breastfeed while undergoing treatment and even three months after receiving the last dose [12]. Currently, the safety and efficacy of luspatercept in children with thalassemia have not been established [12].

Regulatory consideration: Luspatercept was approved by the United States Food and Drugs Administration (US FDA) on November 8, 2019, for use in TDT patients [19-20].

Sotatercept

Sotatercept (ACE-011) is a dimeric recombinant fusion protein that consists of an extracellular domain of the human activin type IIa receptor (ActRIIa) linked to the Fc portion of human immunoglobulin G1 (IgG1) [21-23].

Mechanism of action: Sotatercept acts as a ligand trap and binds activin A and other proteins of the TGF- $\beta$ superfamily that act as negative regulators of late-stage erythropoiesis, preventing their downstream Smad $2 / 3$ signaling and thereby acts to increase mature RBCs [9,22-24].

Pharmacokinetics: Sotatercept in a dose of 0.1 to $1 \mathrm{mg} / \mathrm{kg}$ given subcutaneously follows first-order linear and time-independent pharmacokinetics with an elimination half-life of 21 to 23 days [25].

Clinical trials: A phase 1 study was conducted in a group of 31 healthy volunteers (post-menopausal women) to evaluate the safety, pharmacokinetic, and pharmacodynamic properties of Sotatercept [25]. The drug was administered subcutaneously at a dose of $0.1,0.3$, or $1 \mathrm{mg} / \mathrm{kg}$ every 28 days. A dose-dependent increase was observed in the level of hemoglobin $(\mathrm{Hb})$, hematocrit, and RBC counts; the results were clinically significant and sustained for up to four months.

Another phase 2, open-label, dose-defining, multicenter study attempted to determine the safety and tolerability of Sotatercept in 16 TDT and 30 NTDT adult patients with beta-thalassemia [26]. Transfusion dependence was defined as receiving $\geqslant 2 \mathrm{RBC}$ units every 30 days for $\geqslant 168$ days before study enrollment, with no transfusion-free period of $>45$ consecutive days during this period. Non-transfusion dependence was defined as $\leqslant 1$ transfusion during the 168 days before study enrollment. Sotatercept was given subcutaneously at doses of $0.1,0.3,0.5,0.75$, or $1 \mathrm{mg} / \mathrm{kg}$ every three weeks for $\leqslant 22 \mathrm{months}$, with the median duration of treatment being 13.8 months for TDT patients and 19.6 months for NTDT patients. The study's endpoints were $\mathrm{a} \geqslant 20 \%$ reduction in transfusion burden for 24 weeks in TDT patients and an increase in hemoglobin level of $\geqslant 1.0 \mathrm{~g} / \mathrm{dL}$ for 12 weeks in NTDT patients. Of the 16 TDT patients who received Sotatercept, 10 (63\%) achieved a transfusion burden reduction of $\geqslant 20 \%$ lasting for $\geqslant 24$ weeks; seven (44\%) achieved a reduction of $\geqslant 33 \%$, and two (13\%), a reduction of $\geqslant 50 \%$. The mean change in the Hb level from baseline to the end of treatment was $0.7 \mathrm{~g} / \mathrm{dL}$. In 30 NTDT patients, 18 (60\%) and 11 (37\%) patients achieved a mean $\mathrm{Hb}$ increase of $\geqslant 1.0 \mathrm{~g} / \mathrm{dL}$ and $\geqslant 1.5 \mathrm{~g} / \mathrm{dL}$, respectively, sustained for $\geqslant 12$ weeks. Overall, Sotatercept had a good safety profile, having been tolerated well by most patients.

Dosing: At present, no recommendations exist regarding the optimum dose of Sotatercept. Based on the phase 2 study, a dose of $\geqslant 0.3 \mathrm{mg} / \mathrm{kg}$ and $\geqslant 0.5 \mathrm{mg} / \mathrm{kg}$ given subcutaneously every three weeks is recommended in NTDT and TDT, respectively [22]. 
Adverse effects: Bone pain, arthralgia, back pain, asthenia/fatigue, hypertension, headache, and cough were commonly reported side effects. Injection site erythema, pyrexia, extramedullary hematopoiesis, ventricular extrasystoles, and hypersensitivity were rarely reported. Discontinuation of treatment due to grade 3 or 4 adverse events was not reported. No significant changes in laboratory values for liver and kidney function were noted $[22,24]$.

Regulatory approval: Sotatercept has not yet been licensed for use in beta-thalassemia. More studies are needed to define its position in the treatment algorithm for beta-thalassemia patients. Presently, the drug is not available in the market.

\section{Janus-associated kinase (JAK) inhibitors}

Although the more powerful JAK inhibitors act by aggravating anemia, weak JAK inhibitors may decrease ineffective erythropoiesis. Weak JAK2 inhibitors act by the anti-inflammatory mechanism and induce apoptosis of the more pathological erythroid precursors, thus inducing more efficient erythropoiesis. Past studies on NTDT and TDT animal models revealed that JAK2 inhibition improved ineffective erythropoiesis and reversed splenomegaly, thereby paving a path for JAK1 and JAK2 inhibitors [27-28].

\section{Ruxolitinib}

It is an oral JAK inhibitor that binds and inhibits tyrosine kinases JAK 1 and 2. It is currently approved for polycythemia vera and myelofibrosis, although promising results are emerging in beta-thalassemia [29-30].

Mechanism of action: Ruxolitinib acts on the initial stages of erythropoiesis. Erythropoietin (EPO) binds to its cell surface receptor, activating the cytoplasmic JAK2, which in turn activates downstream pathways involved in erythropoiesis, including the signal transducer and activator of transcription 5 (STAT5), which results in increased proliferation, differentiation, and survival of erythroid precursors [31-32]. Elevated EPO production is seen in animals affected with beta-thalassemia. This pathway is constantly active, leading to extramedullary erythropoiesis and stress erythropoiesis in the spleen [27-28,31].

Pharmacokinetics: Ruxolitinib has an oral bioavailability of $95 \%$, which is not affected by concomitant intake of food, with $97 \%$ of the drug bound to plasma proteins. The peak plasma concentration is achieved one to two hours after administration. It is metabolized primarily by CYP3A4 and to a lesser extent by CYP2C9 and is eliminated by the kidneys. The half-life ranges from three to six hours [33-34].

Clinical trials: A single-arm, multicenter, phase 2 study (TRUTH study) over a span of 30 weeks evaluated the efficacy and safety of ruxolitinib in 30 adult patients with TDT (on regular transfusion regimen requiring $\geqslant 2 \mathrm{RBC}$ units within 4 -week intervals for 24 weeks before enrollment) and splenomegaly $\left(\geqslant 450 \mathrm{~cm}^{3}\right)$ [35]. Ruxolitinib was administered orally starting with a dose of $10 \mathrm{mg}$ twice daily $(5 / 10 \mathrm{mg}$ increments with the maximum dose being $25 \mathrm{mg}$ ) for 30 weeks. A reduction in mean spleen volume of $19.7 \%$ and $26.8 \%$ from baseline at week 12 and week 30, respectively, and about a 6\% reduction in transfused RBC volume was observed. However, no significant improvement in pre-transfusion hemoglobin levels or reduction in the transfusion requirement was seen. An increase in hepcidin levels was seen, although associated significant changes in iron parameters, such as serum iron and ferritin, were not seen. Due to the reasons mentioned above, the study could not proceed to phase 3 [35-36].

Dosing: Currently, there are no dosage recommendations for ruxolitinib in thalassemia.

Adverse effects: The commonly reported side effects were upper respiratory tract infection (27\%), nausea (20\%), upper abdominal pain (17\%), anemia (17\%), diarrhea (17\%), and weight gain (17\%). Serious adverse events reported were anemia, nausea, vomiting, pyrexia, drug-induced liver injury, and pneumonia [35].

Regulatory approval: Ruxolitinib is not approved yet for use in beta-thalassemia.

\section{Iron metabolism modulation}

Hepcidin is the key molecule regulating iron metabolism. There is downregulation of hepcidin in thalassemia due to ineffective erythropoiesis, which potentiates iron overload [7]. Thus, novel therapeutic modalities have been explored to alter iron metabolism and improve erythropoiesis in thalassemia.

Mini Hepcidins

Iron absorption can be reduced by using mini hepcidins. Casu et al. conducted studies in mice models resembling human NTDT and TDT phenotypes and reported an improvement in ineffective erythropoiesis, anemia, a reduction in iron overload, and a decrease in splenomegaly [37-38]. However, two phase 2 trials aimed at assessing the safety and efficacy of mini hepcidins were prematurely terminated due to efficacy issues [39-40]. 


\section{Cureus}

TMPRSS6 and Erythroferrone

TMPRSS6, a serine protease that suppresses hepcidin expression, has also emerged as a promising approach. In a study by Nai et al. in $\mathrm{Hbb}^{\text {th3/+ }}$ mice, TMPRSS6 gene deletion was found to improve anemia, ineffective erythropoiesis, and splenomegaly [41]. A phase 2, multicenter, open-label study by IONIS Pharmaceuticals (Carlsbad, California) is now underway to study the effects of IONIS TMPRSS6-LRx in adult NTDT patients ( $\geqslant 18$ years of age). A single injection of IONIS TMPRSS6-LRx at multiple dose levels will be administered to participants subcutaneously every four weeks for 24 months. The study will measure the increase in hemoglobin from baseline and decrease in liver iron concentration from the baseline in the study participants [42].

In mouse models of beta-thalassemia intermedia, Kautz et al. found that erythroferrone ablation could restore normal levels of hepcidin and suggested that erythroferrone could result in reduced hepcidin production in beta-thalassemia [43]. Smesam et al. conducted a study on 60 TDT patients and compared them with 30 healthy controls [44]. They reported a significant correlation between the reduction in hepcidin levels and an increase in erythroferrone, although no direct correlation of erythroferrone was found with the other measured iron status parameters.

Ferroportin Inhibitors

A novel agent, VIT-2763, which is a small oral molecule, acts as a ferroportin inhibitor. It was found to block iron efflux by competing with hepcidin for ferroportin binding and triggering ferroportin internalization and ubiquitination. In $\mathrm{Hbb}^{\text {th3/+ }}$ mice models, VIT-2763 improved anemia, erythropoiesis, reduced iron overload in the liver, and reduced myelopoiesis in the spleen [45].

A phase 1 double-blind, randomized, placebo-controlled, dose-escalation study was conducted in healthy adult male and female volunteers to determine the safety, tolerability, pharmacokinetic properties, and pharmacodynamic effects of VIT-2763. A temporary decrease in mean serum iron levels and mean transferrin saturation was seen, along with a shift in mean serum hepcidin peaks, after administrating VIT2763 [46]. A randomized, double-blind, placebo-controlled parallel-group trial is currently underway to determine the safety, tolerability, and efficacy of multiple doses of VIT-2763 versus placebo in NTDT patients [47].

Apotransferrin

The administration of apotransferrin (apo-TF) in mice with thalassemic phenotype revealed that apo-TF administration improved ineffective erythropoiesis by increasing the proportion of mature erythroid precursors [48]. Human trials have not been conducted to explore the use of apotransferrin.

\section{Macrophage manipulation therapy}

Although macrophages' interaction with erythrocytes is critical for erythropoiesis, they have also been found to promote stress erythropoiesis, thus limiting sustained erythropoiesis in thalassemia. Ramos et al. used clodronate-loaded liposomes to deplete macrophages in a mouse model of thalassemia [49]. They found that the treatment rapidly ameliorated the anemia and improved erythropoiesis, with a concurrent reduction in spleen size, improved iron status, and a decrease in the $\alpha$-globin chain. This mechanism can also be explored with human trials in the future.

\section{Heat shock proteins (HSP)}

HSP 70, a molecular chaperone, normally protects GATA-1 (a fundamental erythroid transcription factor) from Caspase-3 mediated cleavage during normal terminal erythroid differentiation. This occurs by nuclear accumulation of HSP 70 during terminal maturation [7]. However, HSP70 is sequestrated in the cytoplasm by excess free alpha-globin chains in beta-thalassemia, thereby preventing its nuclear localization and ultimately leading to ineffective erythropoiesis [50]. Therefore, decreasing HSP70 nuclear export (export being usually mediated by exportin 1 , i.e., XPO1) could be a potential therapeutic approach. This was demonstrated by Guillem et al. by using an XPO1 inhibitor to increase the amount of nuclear HSP70, thus rescuing GATA-1 expression and improving terminal differentiation to ameliorate ineffective erythropoiesis in $\beta$-thalassemia [51].

Table 1 provides a snapshot of ongoing/completed clinical trials on the newer pharmacological drugs aimed at improving the ineffective erythropoiesis. 


\section{Cureus}

\begin{tabular}{|c|c|c|c|c|c|c|}
\hline Drug & $\begin{array}{l}\text { Clinical Trial } \\
\text { Identifier }\end{array}$ & $\begin{array}{l}\text { Phase of } \\
\text { Clinical } \\
\text { Trial }\end{array}$ & Status & Location & $\begin{array}{l}\text { Number of } \\
\text { participants }\end{array}$ & Conclusion \\
\hline $\begin{array}{l}\text { Luspatercept } \\
{[12,17]}\end{array}$ & NCT01749540 & II & Completed & Italy & 64 & $\begin{array}{l}\text { Increased hemoglobin concentration, } \\
\text { reduced transfusion burden, and reduced } \\
\text { liver iron content }\end{array}$ \\
\hline Luspatercept [52] & NCT02268409 & $\|$ & Completed & $\begin{array}{l}\text { Italy, } \\
\text { Greece }\end{array}$ & 51 & $\begin{array}{l}\text { Increased hemoglobin concentration and } \\
\text { reduced transfusion burden }\end{array}$ \\
\hline $\begin{array}{l}\text { Luspatercept, } \\
\text { Placebo-controlled } \\
\text { [18] }\end{array}$ & $\begin{array}{l}\text { NCT03342404 } \\
\text { (BEYOND } \\
\text { Trial) }\end{array}$ & $\|$ & $\begin{array}{l}\text { Active, not } \\
\text { recruiting }\end{array}$ & Multinational & 145 & - \\
\hline Luspatercept [53] & NCT04143724 & II & Recruiting & Multinational & 48 & - \\
\hline $\begin{array}{l}\text { Luspatercept, } \\
\text { Placebo-controlled } \\
{[16]}\end{array}$ & $\begin{array}{l}\text { NCT02604433 } \\
\text { (BELIEVE } \\
\text { Trial) }\end{array}$ & III & $\begin{array}{l}\text { Active, not } \\
\text { recruiting }\end{array}$ & Multinational & 336 & Reduced transfusion burden \\
\hline Luspatercept [54] & NCT04064060 & III & Recruiting & Multinational & 665 & - \\
\hline Sotatercept [26] & NCT01571635 & $\|$ & $\begin{array}{l}\text { Active, not } \\
\text { recruiting }\end{array}$ & Multinational & 46 & - \\
\hline Ruxolitinib [36] & $\begin{array}{l}\text { NCT02049450 } \\
\text { (TRUTH Trial) }\end{array}$ & III & Completed & Multinational & 30 & $\begin{array}{l}\text { No change in transfusion requirement, } \\
\text { reduction in spleen volume from baseline } \\
\text { observed }\end{array}$ \\
\hline
\end{tabular}

TABLE 1: Clinical trials for drugs affecting ineffective erythropoiesis in beta-thalassemia patients

Economic considerations: Currently, only Luspatercept (FDA approved) and ruxolitinib (non-FDA approved) are available in the market. Approximately, the price of a vial of $25 \mathrm{mg}$ Luspatercept costs $\$ 3700$, and a vial of $75 \mathrm{mg}$ Luspatercept costs $\$ 11,000$ [55]. Meanwhile, a supply of 60 ruxolitinib tablets of $10 \mathrm{mg}$ costs nearly $\$ 15,000$ [56]. The high costs are a limitation in low and middle-income countries, although if generic versions are made available, the cost can be reduced. This would benefit countries with resource limitations, adding to the spectrum of treatments available for beta-thalassemia.

\section{Conclusions}

Therapeutic management of beta-thalassemia remains a big challenge, especially in low and middle-income countries due to resource limitations. Available treatment options are mainly supportive therapy in the form of regular transfusions and iron chelation. Thus, drugs addressing ineffective erythropoiesis may have a role as adjunctive therapy but at present, recommending the routine use of these novel agents in thalassemia warrants investigations in larger phase 3 trials.

\section{Additional Information}

\section{Disclosures}

Conflicts of interest: In compliance with the ICMJE uniform disclosure form, all authors declare the following: Payment/services info: All authors have declared that no financial support was received from any organization for the submitted work. Financial relationships: All authors have declared that they have no financial relationships at present or within the previous three years with any organizations that might have an interest in the submitted work. Other relationships: All authors have declared that there are no other relationships or activities that could appear to have influenced the submitted work.

\section{References}

1. Galanello R, Origa R: Beta-thalassemia. Orphanet J Rare Dis. 2010, 5:11. 10.1186/1750-1172-5-11

2. Thompson AA, Walters MC, Kwiatkowski J, et al.: Gene therapy in patients with transfusion-dependent $\beta$ thalassemia. N Engl J Med. 2018, 378:1479-93. 10.1056/NEJMoa1705342

3. Gardenghi S, Grady RW, Rivella S: Anemia, ineffective erythropoiesis, and hepcidin: interacting factors in abnormal iron metabolism leading to iron overload in $\beta$-thalassemia. Hematol Oncol Clin North Am. 2010, 24:1089-107. 10.1016/j.hoc.2010.08.003

4. Fibach E, Rachmilewitz EA: Does erythropoietin have a role in the treatment of $\beta$-hemoglobinopathies? . Hematol Oncol Clin North Am. 2014, 28:249-63. 10.1016/j.hoc.2013.11.002 
5. Cappellini MD, Porter JB, Viprakasit V, Taher AT: A paradigm shift on beta-thalassaemia treatment: how will we manage this old disease with new therapies?. Blood Rev. 2018, 32:300-11. 10.1016/j.blre.2018.02.001

6. Bou-Fakhredin R, Tabbikha R, Daadaa H, Taher AT: Emerging therapies in $\beta$-thalassemia: toward a new era in management. Expert Opin Emerg Drugs. 2020, 25:113-22. 10.1080/14728214.2020.1752180

7. Oikonomidou PR, Rivella S: What can we learn from ineffective erythropoiesis in thalassemia? . Blood Rev. 2018, 32:130-43. 10.1016/j.blre.2017.10.001

8. Suragani RN, Cadena SM, Cawley SM, et al.: Transforming growth factor- $\beta$ superfamily ligand trap ACE-536 corrects anemia by promoting late-stage erythropoiesis. Nat Med. 2014, 20:408-14. 10.1038/nm.3512

9. Cappellini MD, Porter J, Origa R, et al.: Sotatercept, a novel transforming growth factor $\beta$ ligand trap, improves anemia in $\beta$-thalassemia: a phase II, open-label, dose-finding study. Haematologica. 2019, 104:477-84. 10.3324/haematol.2018.198887

10. Dussiot M, Maciel TT, Fricot A, et al.: An activin receptor IIA ligand trap corrects ineffective erythropoiesis in $\beta$-thalassemia. Nat Med. 2014, 20:398-407. 10.1038/nm.3468

11. El-Beshlawy A, El-Ghamrawy M: Recent trends in treatment of thalassemia . Blood Cells Mol Dis. 2019, 76:53-8. 10.1016/j.bcmd.2019.01.006

12. Reblozyl ${ }^{\circledR}$ (Luspatercept-aamt). Highlights of prescribing information. (2019). Accessed: July 23, 2021: https://www.accessdata.fda.gov/drugsatfda_docs/label/2019/761136lbl.pdf.

13. Piga A, Perrotta S, Gamberini MR, et al.: Luspatercept improves hemoglobin levels and blood transfusion requirements in a study of patients with $\beta$-thalassemia. Blood. 2019, 133:1279-89. 10.1182/blood-2018-10879247

14. Suragani RN, Cawley SM, Li R, et al.: Modified activin receptor IIB ligand trap mitigates ineffective erythropoiesis and disease complications in murine $\beta$-thalassemia. Blood. 2014, 123:3864-72. 10.1182/blood-2013-06-511238

15. Cappellini MD, Viprakasit V, Taher AT, et al.: A phase 3 trial of Luspatercept in patients with transfusiondependent $\beta$-thalassemia. N Engl J Med. 2020, 382:1219-31. 10.1056/NEJMoa1910182

16. US National Library of Medicine. ClinicalTrials.gov. An efficacy and safety study of luspatercept (ace-536) versus placebo in adults who require regular red blood cell transfusions due to beta ( $\beta$ ) thalassemia. (2020). Accessed: July 23, 2021: https://clinicaltrials.gov/ct2/show/NCT02604433.

17. US National Library of Medicine. ClinicalTrials.gov. Study to evaluate the effects of ace- 536 in patients with beta-thalassemia. (2016). Accessed: July 23, 2021: https://clinicaltrials.gov/ct2/show/NCT01749540.

18. US National Library of Medicine. ClinicalTrials.gov. A study to determine the efficacy and safety of luspatercept in adults with non transfusion dependent beta ( $\beta$ )-thalassemia. (2021). Accessed: July 23, 2021: https://clinicaltrials.gov/ct2/show/NCT03342404.

19. US Food and Drug Administration. FDA approves luspatercept-aamt for anemia in patients with beta thalassemia. (2019). Accessed: July 23, 2021: https://www.fda.gov/drugs/resources-information-approveddrugs/fda-approves-luspatercept-aamt-anemia-patients-beta-th....

20. Businesswire.com. Size F, Wire B. FDA fast track designation granted to luspatercept for the treatment of patients with beta-thalassemia. (2015). Accessed: July 23, 2021:

https://www.businesswire.com/news/home/20150518005252/en/FDA-Fast-Track-Designation-GrantedLuspatercept-Treatment.

21. National Institutes of Health. US National Library of Medicine. Drug Information Portal. Quick access to quality drug information. Sotatercept. (2021). Accessed: July 23, 2021: https://druginfo.nlm.nih.gov/drugportal/name/sotatercept.

22. Cappellini M, Porter J, Origa R, Forni G: A phase 2a, open-label, dose-finding study to determine the safety and tolerability of sotatercept (ACE-011) in adults with beta ( $\beta$ )-thalassemia: interim results. Blood. 2013, 122:3448. 10.1182/blood.V122.21.3448.3448

23. Carrancio S, Markovics J, Wong P, et al.: An activin receptor IIA ligand trap promotes erythropoiesis resulting in a rapid induction of red blood cells and haemoglobin. Br J Haematol. 2014, 165:870-82. 10.1111/bjh.12838

24. Motta I, Bou-Fakhredin R, Taher AT, Cappellini MD: Beta thalassemia: new therapeutic options beyond transfusion and iron chelation. Drugs. 2020, 80:1053-63. 10.1007/s40265-020-01341-9

25. Sherman ML, Borgstein NG, Mook L, et al.: Multiple-dose, safety, pharmacokinetic, and pharmacodynamic study of sotatercept (ActRIIA-IgG1), a novel erythropoietic agent, in healthy postmenopausal women. J Clin Pharmacol. 2013, 53:1121-30. 10.1002/jcph.160

26. US National Library of Medicine. ClinicalTrials.gov: Study to determine the safety and tolerability of sotatercept. (2020). Accessed: July 23, 2021: https://clinicaltrials.gov/ct2/show/NCT01571635.

27. Libani IV, Guy EC, Melchiori L, et al.: Decreased differentiation of erythroid cells exacerbates ineffective erythropoiesis in beta-thalassemia. Blood. 2008, 112:875-85. 10.1182/blood-2007-12-126938

28. Casu C, Presti VL, Oikonomidou PR, Melchiori L, Abdulmalik O, Ramos P, Rivella S: Short-term administration of JAK2 inhibitors reduces splenomegaly in mouse models of $\beta$-thalassemia intermedia and major. Haematologica. 2018, 103:e46-9. 10.3324/haematol.2017.181511

29. Vannucchi AM, Harrison CN: Emerging treatments for classical myeloproliferative neoplasms. Blood. 2017, 129:693-703. 10.1182/blood-2016-10-695965

30. Jakavi-INN-ruxolitinib. Summary of product characteristics. Accessed: July 23, 2021: https://www.ema.europa.eu/en/documents/product-information/jakavi-epar-product-information_en.pdf.

31. Motta I, Scaramellini N, Cappellini MD: Investigational drugs in phase I and phase II clinical trials for thalassemia. Expert Opin Investig Drugs. 2017, 26:793-802. 10.1080/13543784.2017.1335709

32. Melchiori L, Gardenghi S, Rivella S: $\mathbf{X}$-thalassemia: HiJAKing ineffective erythropoiesis and iron overload. Adv Hematol. 2010, 2010:938640. 10.1155/2010/938640

33. Jakafi (Ruxolitinib $\left.{ }^{\circledR}\right)$. (2017). Accessed: July 23, 2021: https://www.accessdata.fda.gov/drugsatfda_docs/label/2017/202192s015lbl.pdf.

34. DrugBank Online. Ruxolitinib. Accessed: July 23, 2021: https://go.drugbank.com/drugs/DB08877.

35. Taher AT, Karakas Z, Cassinerio E, et al.: Efficacy and safety of ruxolitinib in regularly transfused patients with thalassemia: results from a phase 2a study. Blood. 2018, 131:263-5. 10.1182/blood-2017-06-790121 
36. US National Library of Medicine. ClinicalTrials.gov. Study of efficacy and safety of inc 424 in regularly transfused patients with thalassemia. (2017). Accessed: July 23, 2021: https://clinicaltrials.gov/ct2/show/NCT02049450.

37. Casu C, Oikonomidou PR, Chen H, et al.: Minihepcidin peptides as disease modifiers in mice affected by $\beta$ thalassemia and polycythemia vera. Blood. 2016, 128:265-76. 10.1182/blood-2015-10-676742

38. Casu C, Chessa R, Liu A, et al.: Minihepcidins improve ineffective erythropoiesis and splenomegaly in a new mouse model of adult $\beta$-thalassemia major. Haematologica. 2020, 105:1835-44. 10.3324/haematol.2018.212589

39. Clinicaltrials.gov. A study with LJPC-401 for the treatment of myocardial iron overload in adult patients with transfusion-dependent beta thalassemia. (2021). Accessed: July 23, 2021: https://clinicaltrials.gov/ct2/show/NCT03381833.

40. Clinicaltrials.gov. Study of PTG-300 in non-transfusion dependent and transfusion-dependent betathalassemia subjects with chronic anemia (TRANSCEND). (2021). Accessed: July 23, 2021 : https://clinicaltrials.gov/ct2/show/NCT03802201.

41. Nai A, Pagani A, Mandelli G, Lidonnici MR, Silvestri L, Ferrari G, Camaschella C: Deletion of TMPRSS6 attenuates the phenotype in a mouse model of $\beta$-thalassemia. Blood. 2012, 119:5021-9. 10.1182/blood2012-01-401885

42. Study to evaluate the efficacy, safety, tolerability, pharmacokinetics, and pharmacodynamics of IONIS TMPRSS6-LRx. (2019). Accessed: July 23, 2021: https://clinicaltrials.gov/ct2/show/NCT04059406? cond $=$ NCT04059406\&draw $=2$ \&rank $=1$.

43. Kautz L, Jung G, Du X, et al.: Erythroferrone contributes to hepcidin suppression and iron overload in a mouse model of $\beta$-thalassemia. Blood. 2015, 126:2031-7. 10.1182/blood-2015-07-658419

44. Smesam HN, Albuthabhak HA, Arjmand S, Al-Hakeim HK, R Siadat SO: Evaluation of erythroferrone, hepcidin, and iron overload status in Iraqi transfusion-dependent $\beta$-thalassemia major patients. Hemoglobin. 2020, 44:272-7. 10.1080/03630269.2020.1794888

45. Manolova V, Nyffenegger N, Flace A, et al.: Oral ferroportin inhibitor ameliorates ineffective erythropoiesis in a model of $\beta$-thalassemia. J Clin Invest. 2019, 130:491-506. 10.1172/JCI129382

46. Richard F, van Lier JJ, Roubert B, Haboubi T, Göhring UM, Dürrenberger F: Oral ferroportin inhibitor VIT2763: first-in-human, phase 1 study in healthy volunteers. Am J Hematol. 2020, 95:68-77. 10.1002/ajh.25670

47. Clinicaltrials.gov. Safety, tolerability, pharmacokinetics (PK), pharmacodynamics (PD) and preliminary efficacy of VIT-2763 in $\beta$-thalassaemia (VITHAL). (2021). Accessed: July 23, 2021 : https://clinicaltrials.gov/ct2/show/NCT04364269.

48. Li H, Rybicki AC, Suzuka SM, et al.: Transferrin therapy ameliorates disease in beta-thalassemic mice . Nat Med. 2010, 16:177-82. 10.1038/nm.2073

49. Ramos P, Casu C, Gardenghi S, et al.: Macrophages support pathological erythropoiesis in polycythemia vera and $\beta$-thalassemia. Nat Med. 2013, 19:437-45. 10.1038/nm.3126

50. Arlet JB, Ribeil JA, Guillem F, et al.: HSP70 sequestration by free $\alpha$-globin promotes ineffective erythropoiesis in $\beta$-thalassaemia. Nature. 2014, 514:242-6. 10.1038/nature13614

51. Guillem F, Dussiot M, Colin E, et al.: XPO1 regulates erythroid differentiation and is a new target for the treatment of $\beta$-thalassemia. Haematologica. 2020, 105:2240-9. 10.3324/haematol.2018.210054

52. ClinicalTrials.gov. ACE-536 extension study - beta thalassemia . (2021). Accessed: July 23, 2021 : https:/clinicaltrials.gov/ct2/show/NCT02268409?term=luspatercept \&cond=Thalassemia \&draw=2 \&rank=3.

53. US National Library of Medicine. ClinicalTrials.gov. Study of safety \& PK of Luspatercept (ACE-536) in pediatric subjects who require regular RBC transfusions due to beta ( $\beta$ )-thalassemia. (2021). Accessed: July 23, 2021: https://clinicaltrials.gov/ct2/show/NCT04143724.

54. US National Library of Medicine. ClinicalTrials.gov. A study to evaluate long-term safety in subjects who have participated in other luspatercept (ACE-536) clinical trials. (2021). Accessed: July 23, 2021: https://clinicaltrials.gov/ct2/show/NCT04064060.

55. Reblozyl prices, coupons and patient assistance programs. Accessed: September 30, 2021: https://www.drugs.com/price-guide/reblozyl.

56. Jakafi prices, coupons and patient assistance programs. Accessed: September 30, 2021: https://www.drugs.com/price-guide/jakafi. 